\title{
The commonly used plasticizers (bisphenols and phtalates) as endocrine disrupting chemicals in healthy women and women with polycystic ovary syndrome (PCOS).
}

Aleksandra Rutkowska ${ }^{1}$, Aleksandra Konieczna ${ }^{1}$, Kamila Wilczewska², Agnieszka Ciechanowicz², Radosław Czernych ${ }^{4}$, Agnieszka Kowalewska-Włas ${ }^{5}$, Łukasz Łaczmański ${ }^{3}$, Justyna Kuliczkowska ${ }^{3}$, Agnieszka Lenarcik ${ }^{3}$, Anna Trzmiel-Bira ${ }^{3}$, Anna Brona ${ }^{3}$, Monika Kuligowska-Jakubowska ${ }^{1}$, Agata Kot-Wasik ${ }^{2}$, Andrzej Wasik ${ }^{2}$, Andrzej Milewicz ${ }^{3}$, Jacek Namieśnik², Lidia Wolska ${ }^{4}$, Dominik Rachoń ${ }^{1}$

1. Department of Clinical and Experimental Endocrinology, Medical University of Gdansk, Poland

2. Department of Analytical Chemistry, Technical University of Technology of Gdansk, Poland

3. Department and Clinic of Endocrinology, Diabetology and Isotope Therapy, Medical University of Wroclaw, Poland

4. Department of Environmental Toxicology, Medical University of Gdansk, Poland

5. Individual medical practice, Gdansk, Poland

Introduction

Polycystic ovary syndrome (PCOS) is the most common endocrinopathy in women of reproductive age (Azziz, Woods et al. 2004), that lead to fertility problems, type 2 diabetes and coronary heart disease (Mani, Levy et al. 2012).

Plasticizers such as bisphenol A (BPA), bisphenol S (BPS) and phtalates (PAE) are commonly used in daily life in electronic equipment, cans, plastic food containers and bottles. These substances can interact with oestrogen receptors as well as androgen receptors and therefore they are called endocrine disrupting chemicals (EDC). As wehave previouslydescribed (Rutkowska, Rachon, 2014), BPA may play a role in the pathogenesis of PCOS. Briefly, hypothalamic BPA exposure activates GnRH pulse generator, which in turn leads to the exaggerated $\mathrm{LH}$ and decreased FSH production. It can also stimulate androgen production in the ovarian theca cells. All of these effects impair ovarian folliculogenesis leading to anovulation.

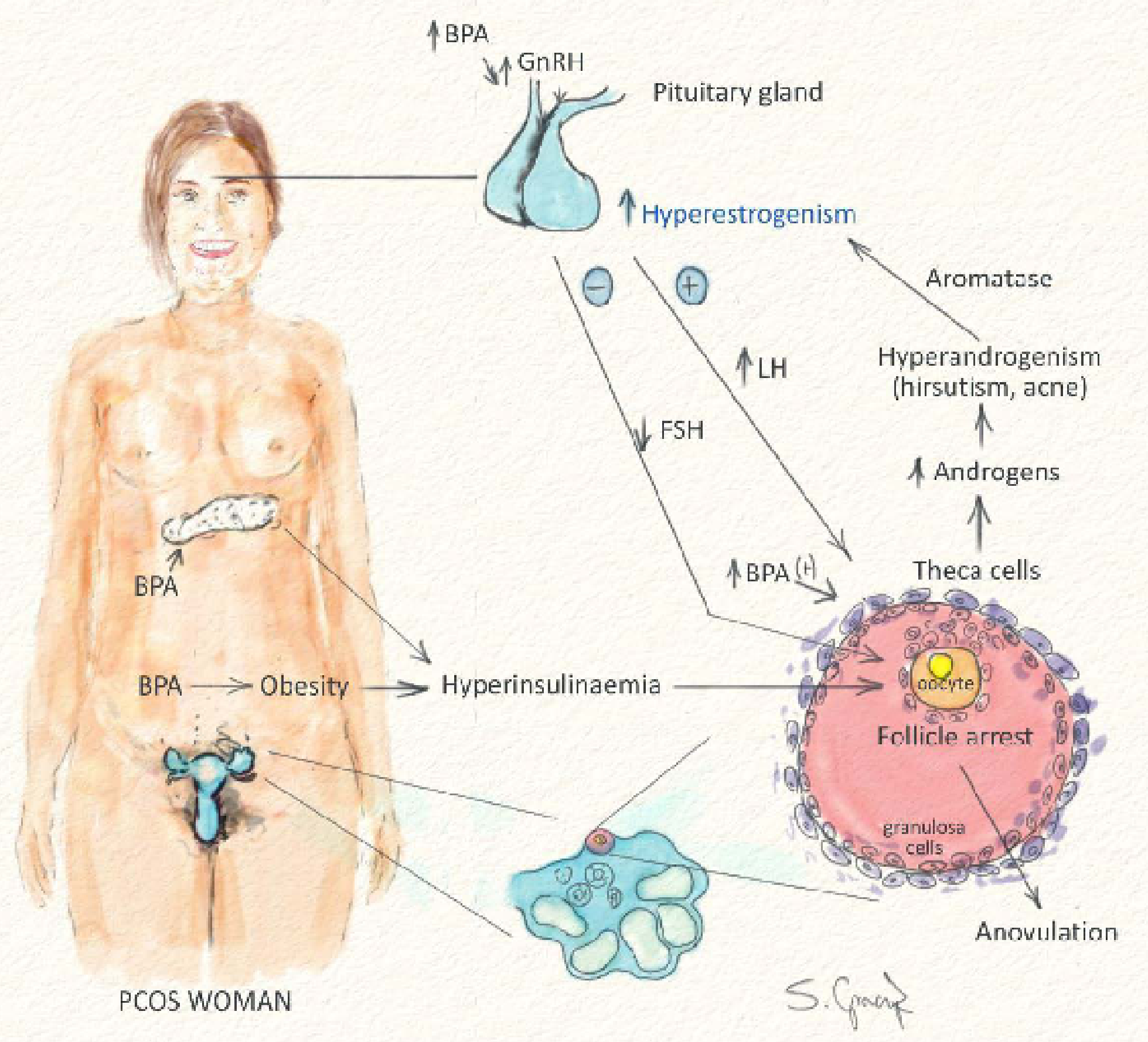

Rutkowska A., Rachoń D., 2014

Aim of study was to evaluate serum concentrations of BPA, BPS and selected PAE in women with PCOS and compare them with healthy controls.

\section{Materials and methods}

In total 133 women were studied. Fifty six patients were diagnosed with PCOS according to the ESHRE/ASRME criteria. The healthy group consisted of 77 women without any endocrinopathy and not taking any hormonal contraceptives. Serum levels of PRL, LH, FSH, 17OH-progesteron, total testosterone, DHEA-S, insulin and SHBG were measured. BPA and BPS concentration were analysed in all women's sera using high pressure liquid chromatography method combined with mass spectrometry. Phtalates concentration (MBP, MEP, MMP, MEHP) were identified using gas chromatography combined with mass spectrometry.

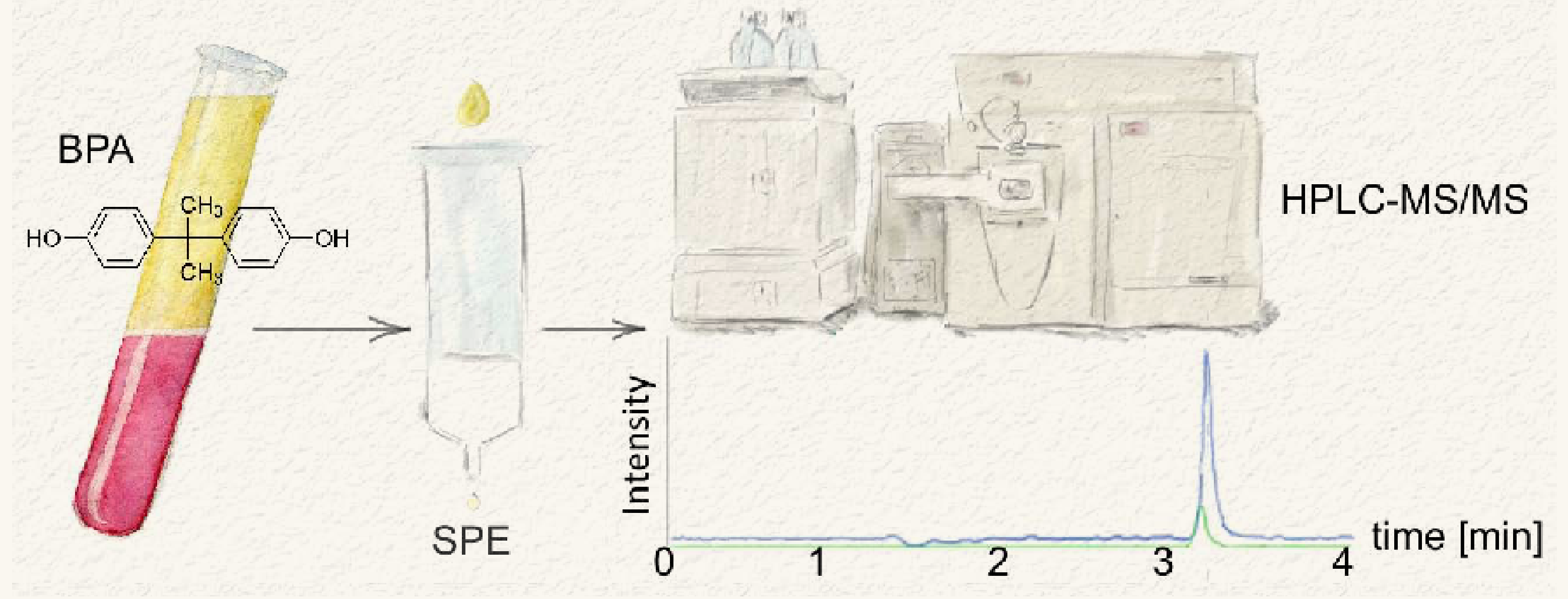

Results

Clinical characteristics of the studied women

\begin{tabular}{|l|c|c|c|}
\hline & $\begin{array}{c}\text { CONTROL } \\
(\mathbf{N}=\mathbf{7 7})\end{array}$ & $\begin{array}{c}\text { PCOS } \\
(\mathbf{N}=56)\end{array}$ & $\mathbf{p}$ \\
\hline Age (yrs) & $26,9 \pm 6,61$ & $26,31 \pm 5,39$ & 0,63 \\
\hline BMI & $21,88 \pm 1,2$ & $21,92 \pm 1,37$ & 0,84 \\
\hline Total testosterone [nmol/I] & $1,29 \pm 0,54$ & $1,61 \pm 0,69$ & 0,044 \\
\hline FAl & $2,15 \pm 1,65$ & $3,24 \pm 1,99$ & 0,002 \\
\hline $17 \beta$-estradiol [pg/ml] & $159,53 \pm 189,43$ & $127,67 \pm 137,11$ & 0,1 \\
\hline LH/FSH & $1,2 \pm 0,82$ & $1,69 \pm 1,07$ & 0,0014 \\
\hline SHBG [nmol/I] & $65,79 \pm 33,11$ & $63,5 \pm 35,21$ & 0,87 \\
\hline
\end{tabular}

\begin{tabular}{|l|c|c|c|}
\hline $\begin{array}{l}\text { Serum concentrationsof } \\
\text { the selected EDC }[\mathbf{n g} / \mathbf{m l}]\end{array}$ & $\begin{array}{c}\text { CONTROL } \\
(\mathbf{N}=\mathbf{7 7})\end{array}$ & $\begin{array}{c}\mathbf{P C O S} \\
\mathbf{( N = 5 6 )}\end{array}$ & $\mathbf{p}$ \\
\hline Bisphenol A (BPA) & $12,86 \pm 7,99$ & $19,43 \pm 13,98$ & 0,03 \\
\hline Monobutyl phtalate (MBP) & $0,267 \pm 0,202$ & $0,655 \pm 0,690$ & 0,07 \\
\hline Monoethyl phtalate (MEP) & $0,064 \pm 0,02$ & $0,089 \pm 0,12$ & 0,64 \\
\hline $\begin{array}{l}\text { Monomethyl phtalate } \\
\text { (MMP) }\end{array}$ & $0,053 \pm 0,031$ & $0,022 \pm 0,013$ & 0,03 \\
\hline $\begin{array}{l}\text { Mono(2-ethylhexyl) phthal- } \\
\text { ate (MEHP) }\end{array}$ & $0,698 \pm 0,488$ & $0,647 \pm 0,628$ & 0,5 \\
\hline
\end{tabular}

Correlations between serum BPA concentrations and hormonal profile of women with PCOS
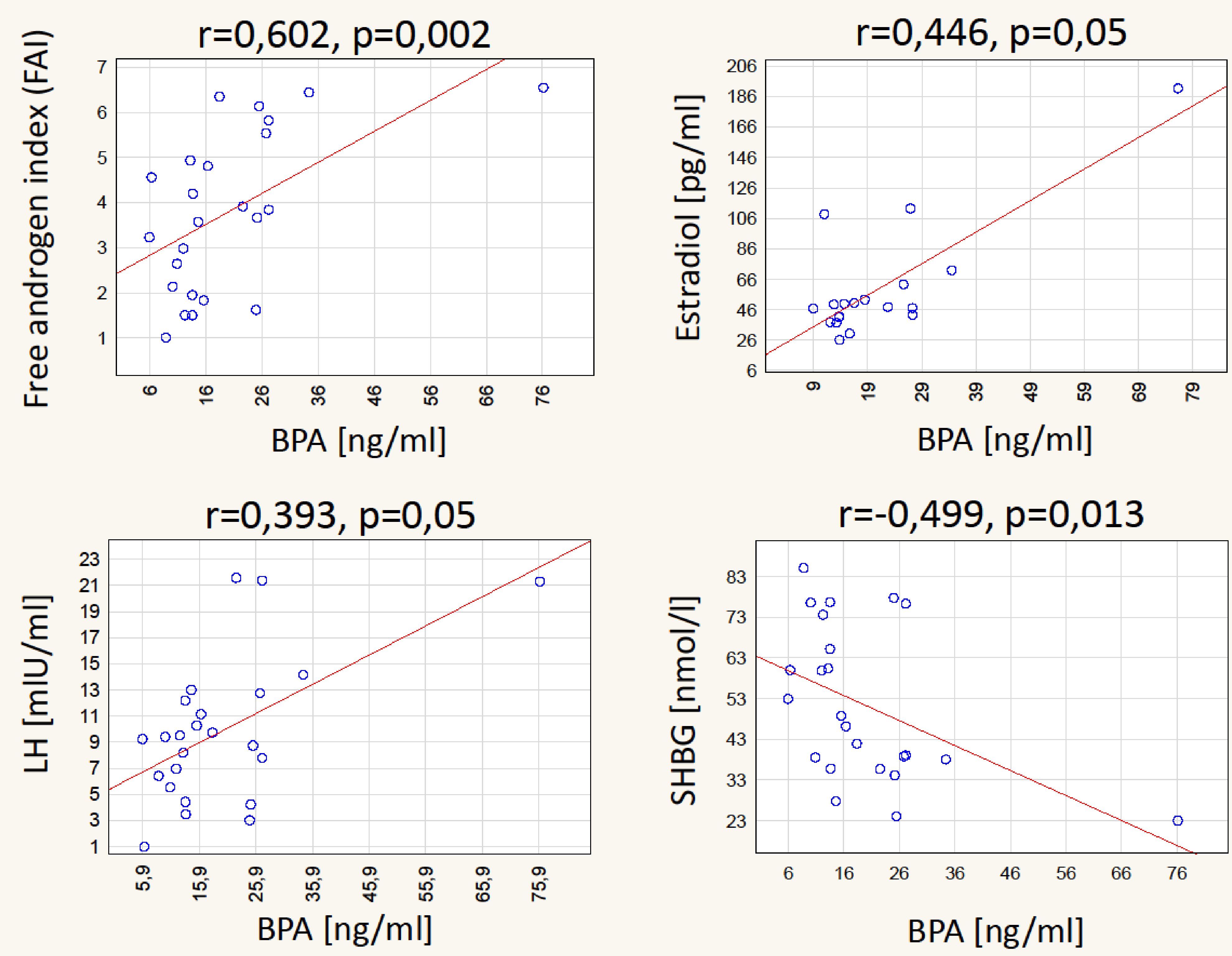

Correlations between selected serum PAE concentrations and hormonal profile of women with PCOS
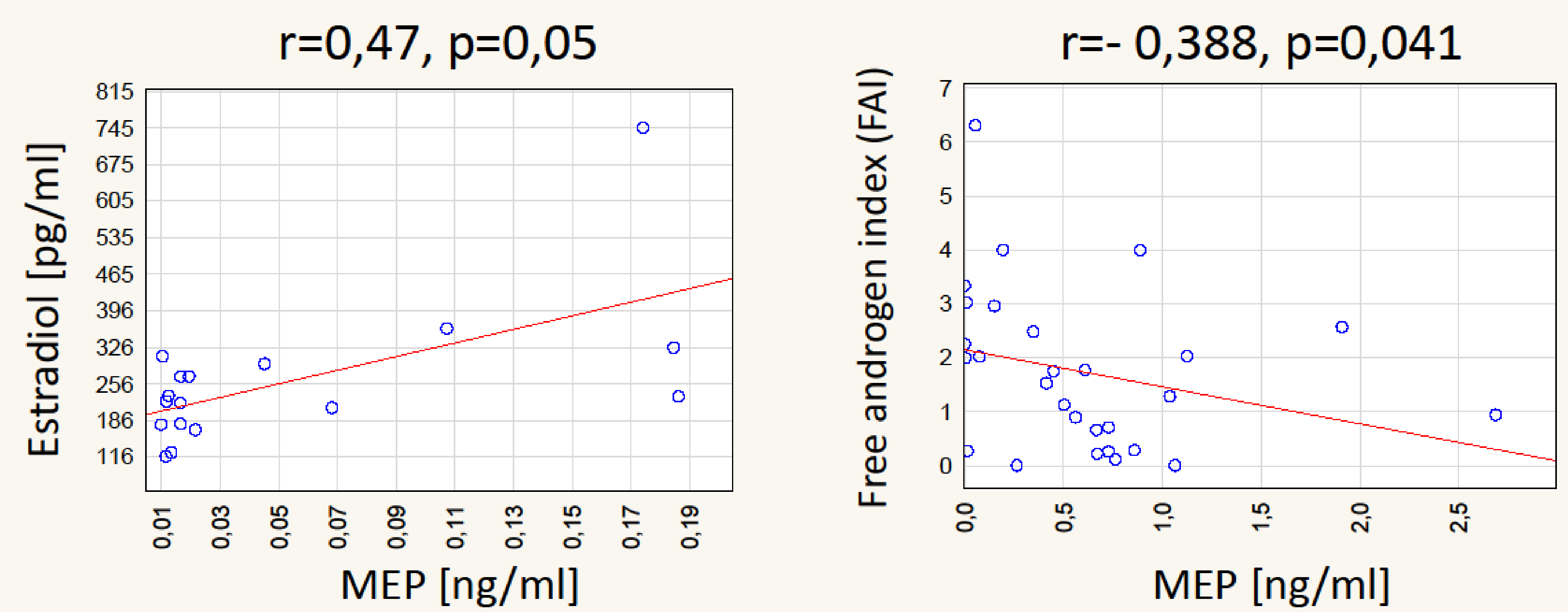

Results summary

In our project with discovered:

1. Significantly higher serum BPA and MBP concentrations in women with PCOS.

2. Positive correlations between serum BPA concentration and FAI, estradiol and LH in women with PCOS.

3. Negative correlation between serum BPA concentration and SHBG level in women with PCOS.

4. Positive correlation between serum MEP concentration and estradiol level and negative correlation between MEHP and FAI.

5. No correlation between BPA and PAE concentrations with hormonal profile of healthy women, except of MBP and FAI $(r=0,73, p=0,024)$

\section{Conclusions}

These results confirm the hypothesis of endocrine disrupting potential of plasticizers in women with PCOS and suggest the need of biomonitoring of these chemicals in the environment and human biological samples.

\section{REFERENCES:}

Azziz, R., K. S. Woods, et al. (2004). "The prev

J Clin Endocrinol Metab 89(6): 2745-2749. Mani, H., M.J. Levy, et al. (2013). "Diabetes and cardiova

spective cohot study. Cin Endochinol(Ox). 78(6):926-3 Rutkowska, A., Rachoń, D (2014). „Bisphenol A (BPA) an

(PCOS)". Gynecol Endocrinol. 2014; 30(4):260-265.

The project was partially financed by Ministry of Science and Higher Education (grant No: MN 01-0156/08).

Address of correspondence: Aleksandra Rutkowska, Department of Clinical and Experimental Endocrinology, Medical University of Gdansk, Dębinki 1, 80-211, Gdańsk, Poland; mail: olarynio@gumed.edu.pl 